\title{
Solid Cystic Pseudopapillary Tumor of the Pancreas in Children: A Case Series
}

\author{
Evangeline P. Capul, MD, ${ }^{1}$ Germana V. Gregorio, $\mathrm{MD}^{1}$ and Jose Ma. C. Avila, MD² \\ ${ }^{1}$ Section of Pediatric Gastroenterology, Hepatology and Nutrition, Department of Pediatrics Philippine General Hospital University of the Philippines Manila \\ ${ }^{2}$ Department of Pathology Philippine General Hospital College of Medicine, University of the Philippines Manila
}

\begin{abstract}
Solid cystic pseudopapillary tumor (SCPT) of the pancreas is rare and is difficult to diagnose pre-operatively. We describe four children with abdominal pain and abdominal mass who were diagnosed with SCPT. Three underwent resection of the mass, one an open liver biopsy. Histopathology showed solid epithelioid cells, cystic areas, and pseudopapillary structures. SCPT is a low-grade malignant tumor with good prognosis and should be suspected in any child with an abdominal mass and abdominal pain.
\end{abstract}

Key Words: Solid cystic pseudopapillary tumor, pancreas

\section{INTRODUCTION}

Solid cystic pseudopapillary tumor of the pancreas $(\mathrm{SCPT})$ is a rare pancreatic neoplasm first described by Frantz on four cases and initially referred to as "papillary tumor of the pancreas." Literature has referred to this tumor using a number of terminologies including: Frantz or Hamoudi tumor, Gruber - Frantz's tumor, adenocarcinoma of the pancreas in childhood, solid and papillary tumor, papillary cystic tumor or carcinoma, papillary cystic epithelial neoplasm, papillary and solid neoplasm, solid and cystic acinar tumor, solid and cystic papillary tumor, papillary and cystic epithelial carcinoma, solid cystic papillary epithelial neoplasm, and solid pseudopapillary tumor or carcinoma. ${ }^{1,2,3}$ Among these terms, solid cystic pseudopapillary tumor best describes the pathologic features as grossly, the tumor showed solid tissue surrounding central hemorrhagic and cystic area, and histologically, there is note of variegated formation of solid, pseudopapillary, and cystic patterns. ${ }^{1}$

SCPT can occur anywhere in the pancreas and usually exhibits exophytic growth. ${ }^{2}$ They are frequently localized in the pancreatic tail or head, and only $1 \%$ have extrapancreatic localization. ${ }^{1}$

In a study by Guo of 24 cases, $33.3 \%$ is located in the tail, $29.2 \%$ in the head, $12.5 \%$ in head and body as well as body and tail, $8.3 \%$ in the body and $4.2 \%$ in the processus anconaeous. ${ }^{4}$

The incidence of SCPT of the pancreas is unknown. It has been reported that SCPT accounts for 1 to $2 \%$ of exocrine pancreatic tumors, usually occurring among young females aged 19-50 years. Less than 10\% of them are males and are generally ten years older than women. SCPT of the pancreas is rare in children ${ }^{2}$ with only 48 cases reported in 
children with an average age of 11 years (range: 8-16 years) and a male: female ratio of $1: 5 .^{5}$

There is presently no local study on solid cystic pseudopapillary tumor of the pancreas in children. This case series will describe four pediatric cases diagnosed at the Philippine General Hospital for three years (2012-2014). Their clinical features, imaging studies, histopathologic findings, and surgical interventions will also be discussed. Parents were verbally informed and agreed that the data of the patients will be included in this case series of patients with SCPT.

\section{CASE 1}

A 7-year-old male is admitted for a two-year history of vague abdominal pain, non-bilious vomiting, and palpable mass at the right lower quadrant, which was hard, nodular, non-movable, non-tender and measured $14 \mathrm{~cm} \times 8 \mathrm{~cm}$. CT scan of the abdomen confirmed the presence of a large, lobulated heterogeneous enhancing intraabdominal mass (13 $\mathrm{cm} \times 14 \mathrm{~cm} \times 14 \mathrm{~cm}$ ) with cystic and solid areas deflecting the liver superiorly (Figure 1). Exploratory laparotomy with open liver biopsy was done, and histopathology revealed solid cystic pseudopapillary neoplasm of the pancreas (Figure 2). The parents refused resection of the mass. In the interim of three years, there was the persistence of abdominal pain.

Three weeks before admission, there was a note of anorexia, weight loss, undocumented fever, abdominal distension, and bilious vomiting. A repeat CT scan disclosed that the pancreatic mass was bigger $(27 \mathrm{~cm} \times 23 \mathrm{~cm} \mathrm{x}$ $11 \mathrm{~cm}$ ), deflecting the liver superiorly with enhancing foci in the liver and multiple lymph nodes at the aortocaval, perisplenic, perigastric, and inguinal regions. Another surgical resection was contemplated but parents opted that no further intervention and went home per request.
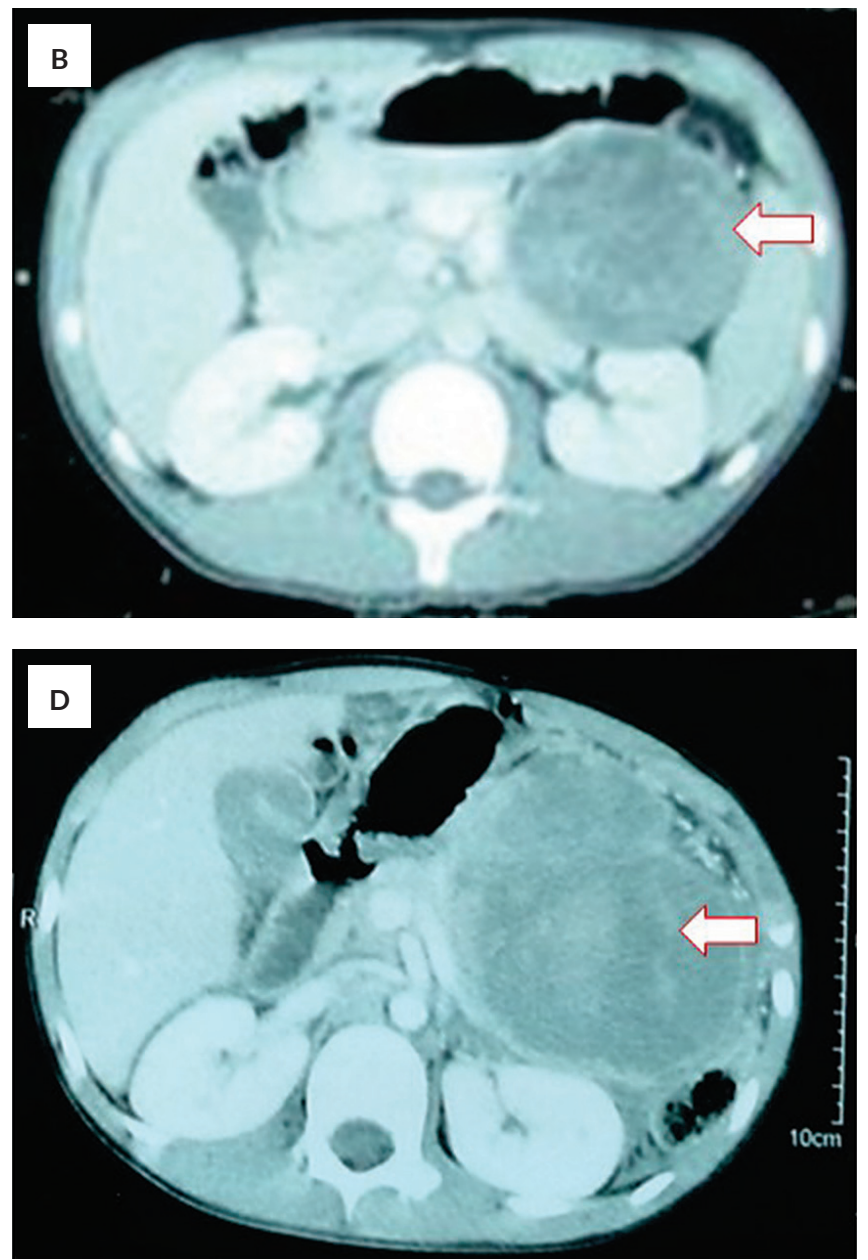

Figure 1. CT scan of four patients diagnosed with SCPT. (A) Case 1: Large, lobulated heterogeneous enhancing intraabdominal mass $(13 \mathrm{~cm} \times 14 \mathrm{~cm} \times 14 \mathrm{~cm}$ ) with cystic and solid areas deflecting the liver superiorly. (B) Case 2: Well-defined, ovoid, heterogeneous, hypo-enhancing focus $(8 \mathrm{~cm} \times 7 \mathrm{~cm} \times 7 \mathrm{~cm})$ with few thin internal septations centered in the left anterior perirenal space. (C) Case 3: Fairly-defined mixed attenuating, heterogeneously-enhancing focus $(11 \mathrm{~cm} X 11 \mathrm{~cm} X 10 \mathrm{~cm})$ with hypodense regions that may represent areas of necrosis. (D) Case 4: Heterogenous lobulated mass in the left upper and mid-abdomen. 

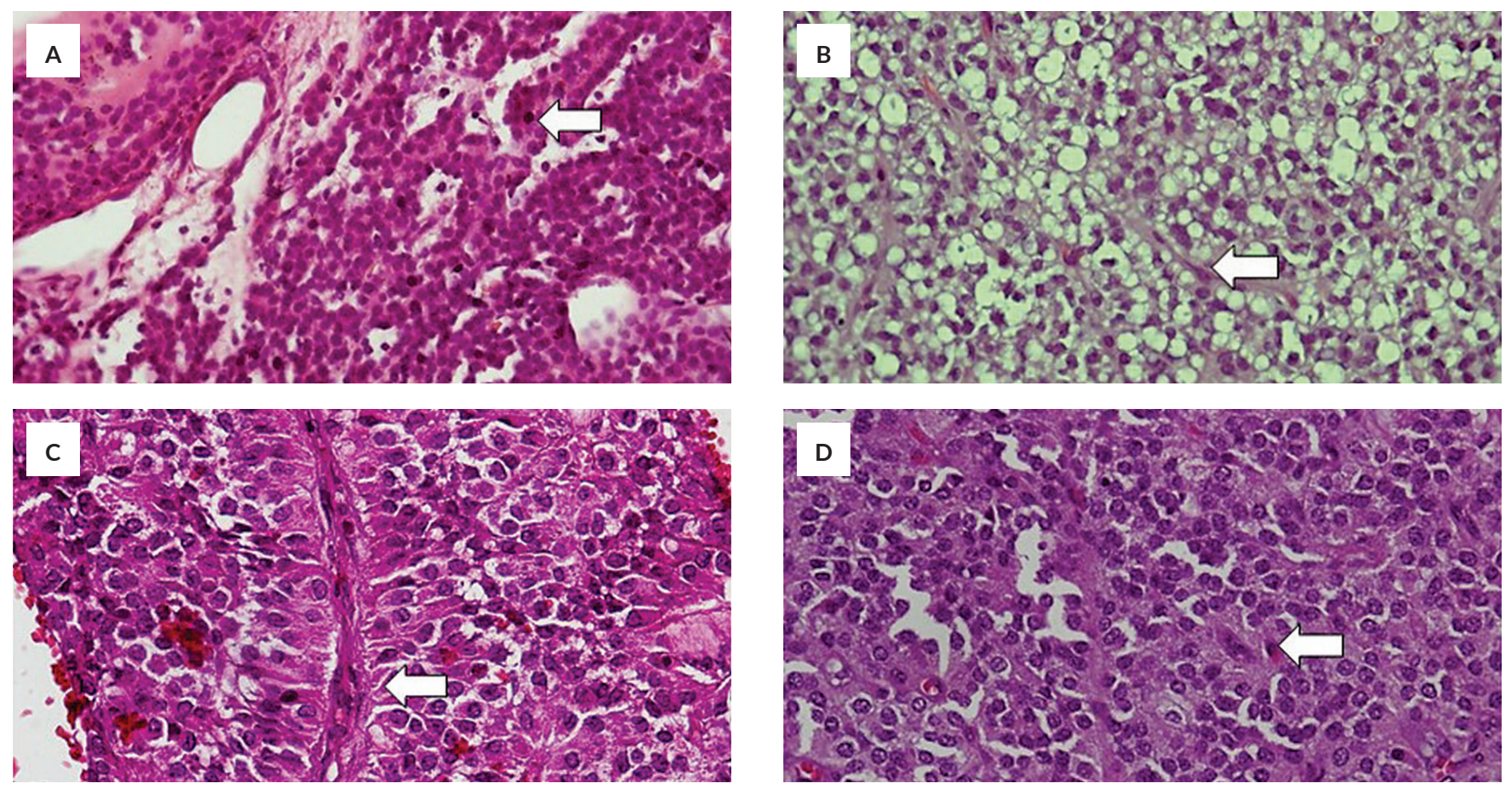

Figure 2. Histopathology results of four patients diagnosed with SCPT. (A) Case 1: Cells with a vesicular round to oval nuclei with fine and pepper chromatin pattern with ample eosinophilic granular cytoplasm (H\&E 40X). (B) Case 2: Regions of solid, pseudopapillary, and cystic changes merging with one another (H\&E 40X). (C) Case 3: Papillary structures with vascular cores covered by several layers of bland tumor cells (H\&E 40X). (D) Case 4: Pseudopapillary regions and cells with round nuclei and eosinophilic cytoplasm (H\&E 40X).

\section{CASE 2}

A 12-year-old male consulted with a one-month history of left upper quadrant pain, characterized as persistent and dull associated with anorexia and post-prandial vomiting occurring 3-4 times a day. Increased severity of the pain prompted consult. Pertinent physical examination revealed a soft flat abdomen with a firm, well-defined mass $5 \mathrm{~cm} \mathrm{x} 5$ $\mathrm{cm}$ in the left upper quadrant with slight tenderness on deep palpation. It was confirmed on a CT scan of the abdomen as a well-defined, ovoid, heterogeneous, hypo-enhancing focus $(8 \mathrm{~cm} \times 7 \mathrm{~cm} \times 7 \mathrm{~cm}$ ) with few thin internal septations centered in the left anterior perirenal space continuous with the pancreatic tail anteromedially (Figure 1). An exploratory laparotomy was done, which showed a $7 \mathrm{~cm} \mathrm{x}$ $6 \mathrm{~cm}$ cystic mass with solid components at the mesentery at the left upper quadrant (Figure 3). Excision of mesenteric cyst with biopsy and Jackson Pratt drain insertion was performed. Histopathology is consistent with solid cystic pseudopapillary tumor (Figure 2).

\section{CASE 3}

A 16-year-old female consulted due to a sixmonth duration of intermittent abdominal pain, which spontaneously resolves with a note of a fist-size mass at the right upper quadrant. There was no associated bowel movement changes, vomiting, anorexia, and early satiety. No consult was done until a few days before admission, when there was a note of severe right upper quadrant pain, boring in character, radiating to the back. The abdomen is soft and flat on physical examination, with normoactive bowel sounds, intact Traube's space and no fluid wave. A nodular non-movable mass measuring $8 \mathrm{~cm}$ x $10 \mathrm{~cm}$ was palpated at the epigastric area. CT scan of the abdomen exhibited a fairly-defined mixed attenuating, heterogeneously-enhancing focus $(11 \mathrm{~cm} \times 11 \mathrm{~cm} \times 10 \mathrm{~cm})$ from the stomach wall with hypodense regions. The pancreas is of average size and tissue homogeneity (Figure 1).

Exploratory laparotomy revealed a pancreatic head mass $14 \mathrm{~cm} \times 12 \mathrm{~cm}$, predominantly cystic, adherent, and pushing the duodenum anteriorly, common bile duct laterally, transverse colon inferiorly, and the stomach laterally and superiorly (Figure 3). Paraaortic lymph nodes were not noted. Whipple procedure (pancreaticoduodenectomy) was performed to facilitate the resection of the mass. Histopathology demonstrated regions of solid, pseudopapillary, and cystic changes merging with one another, suggestive of solid pseudopapillary cystic tumor of the pancreas (Figure 2). The margins of resection and nodes were also negative for tumor. Immunohistochemistry staining was positive for vimentin and negative for chromogranin. 

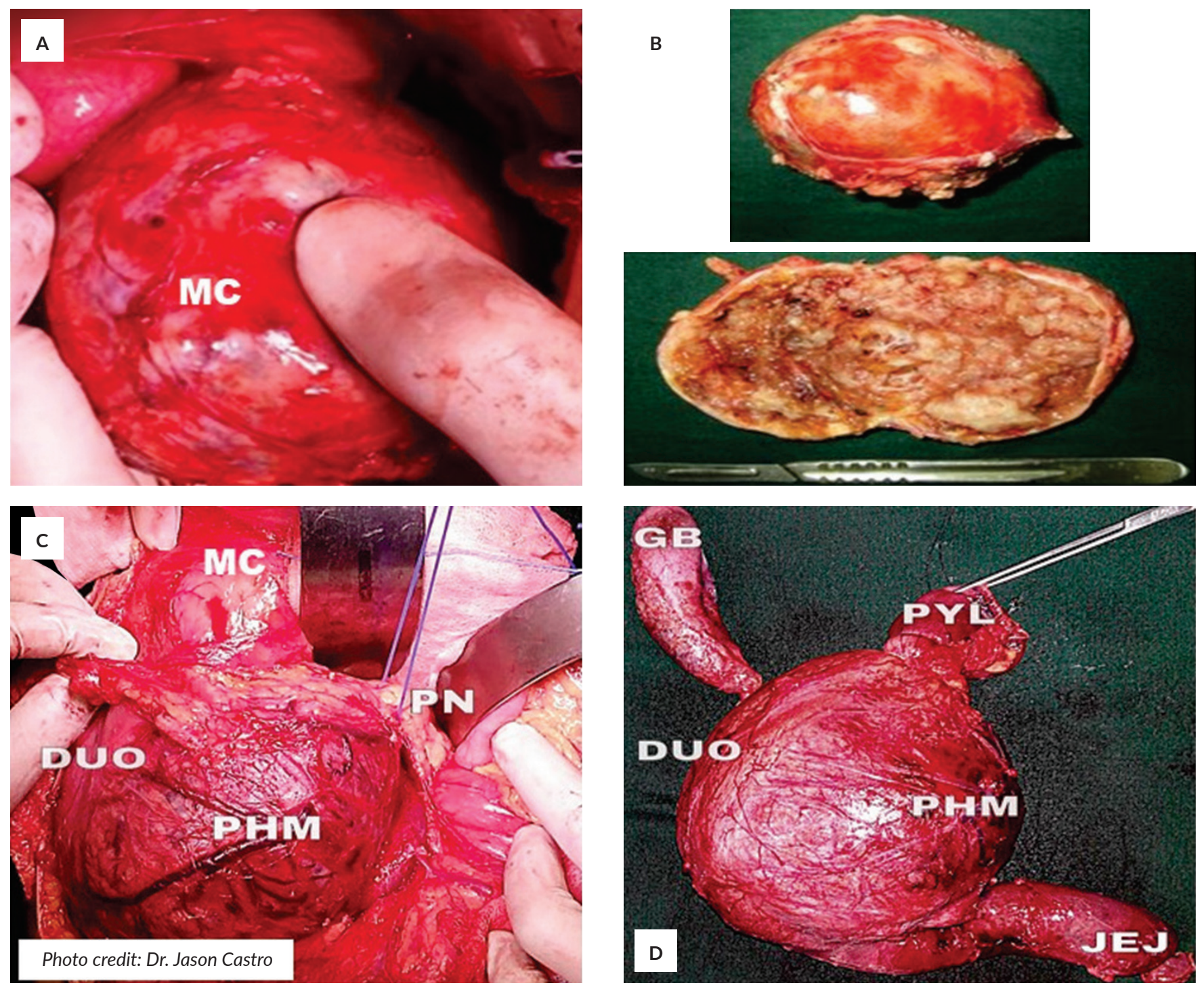

Figure 3. Intraoperative findings of SCPT patients - Case 2: (A) Cystic mass $(7 \mathrm{~cm} \mathrm{X} 6 \mathrm{~cm})$ with solid components at the mesentery at the left upper quadrant. (B) Cut-section of the cystic mass. Case 3: (C) Predominantly cystic pancreatic head mass $(14 \mathrm{~cm} \times 12 \mathrm{~cm})$, adherent, and pushing the duodenum anteriorly. (D) Pancreatic head mass.

[MC-mesenteric cyst, DUO-duodenum, PHM-pancreatic head mass, PN-pancreatic neck, GB-gall bladder, PYL-pylorus, JEJ-jejunum]

\section{CASE 4}

A 9-year-old male whose history revealed that he was kicked in the abdomen one year ago and developed intraabdominal hematoma and hemoperitoneum, which responded with medical decompression. A ball hit him in the abdomen for this present illness, and immediate consult was done as a LUQ mass was palpated. On physical examination, he was noted to be stunted but not wasted. The abdomen was soft, globular, with a tender $5 \mathrm{~cm} \times 5 \mathrm{~cm}$ mass at the left upper quadrant with spleen palpable $4 \mathrm{~cm}$ below the left costal margin. CT scan of the abdomen showed a heterogeneous lobulated mass in the left upper and midabdomen; considerations include hematoma, abscess, or a neoplastic process (Figure 1). He underwent exploratory laparotomy with excision of pancreatic mass at the body (9 $\mathrm{cm} \times 8 \mathrm{~cm} \times 6 \mathrm{~cm}$ ). The spleen was enlarged, and there was the presence of perigastric and peripancreatic lymph nodes. Histopathology was suggestive of solid cystic pseudopapillary tumor of the pancreas (Figure 2).

\section{DISCUSSION}

Our case series documents four Filipino children with solid cystic pseudopapillary tumors, a rare pancreatic tumor previously reported in only 48 children worldwide, the majority from Korea. However, it has also been described in other Asian, South American, and European countries. 
It is a disease predominantly seen among younger females, aged 8-16 years, although three of the four cases were males in our present study.

SCPT is challenging to detect pre-operatively, and the final diagnosis is only made once the characteristic histopathologic appearance of solid, cystic, and pseudopapillary pattern is demonstrated. The most common clinical presentation is the presence of non-specific abdominal pain with or without a palpable abdominal mass, as what we have demonstrated in our patients (Table 1). Associated nausea and vomiting symptoms may be caused by the compression of the stomach and other adjacent organs. Rarely, the patient may be asymptomatic. ${ }^{6}$ There was a history of abdominal trauma in one of our patients (case 4), but this has not been associated with the pathologic development of SCPT.
Differential diagnosis of SCPT includes injury-related such as pseudo- and infection-related cyst; congenital cyst such as duplication (heterogeneous) cyst and duodenal diverticula; neoplastic cyst such as serous/mucinous cyst; and other disorders such as mature cystic teratoma, lymphoepithelial cyst, squamous cyst of the pancreatic duct, cystic hamartoma, endometriotic cysts and metastatic ovarian and renal cell carcinoma.

There are no specific biochemical abnormalities that will suggest the disease. Amylase and lipase, which are usually employed to assess pancreatic involvement, are usually normal as what we have observed. Similarly, patients at diagnosis have acceptable levels of tumor markers for pancreatic (CA 19-9), hepatic (alpha-fetoprotein), and colonic (carcinoembryonic antigen) markers as detected in

Table 1. Summary of Four Cases with Solid Cystic Pseudopapillary Tumor

\begin{tabular}{|c|c|c|c|c|}
\hline & Case 1: 7 year old/M & Case 2: 12-year-old/M & Case 3: 16-year-old/F & Case 4: 9-year-old/M \\
\hline Chief Complaint & Abdominal pain & Abdominal pain & Abdominal pain & Abdominal pain \\
\hline Duration of illness & 24 months & 1 month & 6 months & $\begin{array}{l}\text { A few hours prior } \\
\text { to consult }\end{array}$ \\
\hline \multicolumn{5}{|l|}{ Signs and symptoms } \\
\hline Abdominal pain & + & + & + & + \\
\hline Palpable abdominal mass & + & + & + & + \\
\hline Vomiting & + & + & - & + \\
\hline Anorexia & + & + & - & - \\
\hline CT Scan assessment & $\begin{array}{l}\text { First (5/19/2011): Large, intra- } \\
\text { abdominal enhancing mass with } \\
\text { cystic and solid areas, deflecting the } \\
\text { surrounding abdominal structures, } \\
\text { primary consideration is hemangio- } \\
\text { lymphangioma complex, but cannot } \\
\text { rule out a malignant process } \\
\text { Second (12/10/13): Pancreas converted } \\
\text { into a large, well-defined, lobulated } \\
\text { predominantly solid, mixed-attenuating, } \\
\text { heterogeneously-enhancing mass at } \\
\text { the right abdominopelvic region }\end{array}$ & $\begin{array}{l}\text { Large pancreatic focus } \\
\text { for which the primary } \\
\text { consideration is a } \\
\text { pancreatic pseudocyst. } \\
\text { Differential diagnoses } \\
\text { include pancreatic } \\
\text { abscess and mucinous } \\
\text { cystic pancreatic tumor }\end{array}$ & $\begin{array}{l}\text { Exophytic gastric } \\
\text { mass, considerations } \\
\text { include gastro- } \\
\text { intestinal stromal } \\
\text { tumor and leiomyoma }\end{array}$ & $\begin{array}{l}\text { Heterogenous } \\
\text { lobulated mass in } \\
\text { the left upper and } \\
\text { mid-abdomen. } \\
\text { Considerations } \\
\text { include hematoma, } \\
\text { abscess, or neo- } \\
\text { plastic process. }\end{array}$ \\
\hline $\begin{array}{l}\text { Radiographic description of } \\
\text { the pancreas }\end{array}$ & $\begin{array}{l}\text { First (5/9/2011): } \\
\text { Normal in size and configuration. } \\
\text { Second (12/10/13): Pancreas is a } \\
\text { large, well-defined, heterogeneously- } \\
\text { enhancing mass }\end{array}$ & $\begin{array}{l}\text { Large, well-defined, } \\
\text { ovoid, heterogeneous } \\
\text { mass is continuous } \\
\text { with the pancreatic tail } \\
\text { and body. }\end{array}$ & $\begin{array}{l}\text { The pancreas is of } \\
\text { normal size and } \\
\text { tissue homogeneity. } \\
\text { The pancreatic duct } \\
\text { is not dilated. }\end{array}$ & Not specified \\
\hline Surgical intervention & $\begin{array}{l}\text { Exploratory laparotomy (EL), an open } \\
\text { biopsy of the mass. }\end{array}$ & $\begin{array}{l}\text { EL, excision of the } \\
\text { mesenteric cyst with } \\
\text { biopsy and Jackson } \\
\text { Pratt drain insertion }\end{array}$ & $\begin{array}{l}\text { EL, Whipple } \\
\text { procedure }\end{array}$ & $\begin{array}{l}\text { EL, excision of } \\
\text { pancreatic mass }\end{array}$ \\
\hline Intraoperative findings & & $\begin{array}{l}7 \mathrm{~cm} \times 6 \mathrm{~cm} \text { cystic mass } \\
\text { with solid components } \\
\text { at the mesentery } \\
\text { (left upper quadrant) }\end{array}$ & $\begin{array}{l}15 \mathrm{~cm} \times 10 \mathrm{~cm} \\
\text { pancreatic head mass }\end{array}$ & $\begin{array}{l}\text { Pancreatic mass } \\
\text { at the body } \\
9 \mathrm{~cm} \times 8 \mathrm{~cm} \times 6 \mathrm{~cm}\end{array}$ \\
\hline \multicolumn{5}{|l|}{ Tumor markers } \\
\hline $\begin{array}{l}\text { AFP [Normal value: } \\
1.08-8.041 \mathrm{U} / \mathrm{mL}]\end{array}$ & $(12 / 11 / 2013)>500$ & 1.37 & ND & ND \\
\hline $\begin{array}{l}\text { CA } 19-9 \text { [Normal value: } \\
\text { 0-37.0U/mL] }\end{array}$ & ND & 14.95 & 43.5 & ND \\
\hline \multicolumn{5}{|l|}{ Immunohistochemistry } \\
\hline Vimentin & - & ND & + & + \\
\hline Chromogranin & - & ND & - & - \\
\hline Cytokeratin & - & - & ND & ND \\
\hline
\end{tabular}

ND - Not done 
one of our cases (case 2) (Table 1). Our first case had an elevated alpha-fetoprotein during the second admission. It was also noted that there were already multiple enhancing foci in the liver, suggestive of metastases.

Characteristics of imaging studies usually include large, round, well-encapsulated, combined cystic, and solid masses in the pancreas. Calcifications or internal septations may also be detected in some cases. All our patients underwent abdominal ultrasonography, which was eventually followed by computed tomography of the abdomen. In only one (case 2) of the four patients was the mass localized in the pancreas, which was interpreted as a pancreatic pseudocyst. In other cases, the CT scan findings suggested the presence of an intrabdominal mass, gastric tumor, and hematoma. Had the diagnosis of SCPT been suspected, magnetic resonance imaging (MRI) would have assisted in making the diagnosis. MRI is superior compared to CT scan in distinguishing specific tissue characteristics of SCPT, such as hemorrhage, cystic degeneration, or the presence of a capsule. ${ }^{5}$

The histological appearance of SCPT is distinctive and diagnostic, as demonstrated in all the biopsies of our cases. The tumor's solid components are composed of sheets and nests of uniform, polygonal epithelioid cells arranged around a network of small, delicate blood vessels, surrounded by varying amounts of collagen. The cystic areas are attributed to the presence of hemorrhage and necrosis. Extensive degenerative changes in the tumor may eventually develop into pseudopapillary structures. ${ }^{5}$

Immunohistochemical staining is done to differentiate SCPT from other pancreatic neoplasms, but only selected stains were facilitated in our patients due to the prohibitive cost. Neuron-specific enolase and carcinoembryonic antigen and CA 19-9, which are positive for acinar cell carcinoma and pancreatic adenoma, were not done as these tumors are very rare in children. Two of our patients (cases 3 and 4) stained positive for vimentin, a marker of mesenchymal derived cells and is positive in 90\% of SCPT. Both cases, however, were negative for chromogranin, which is a marker for neuroendocrine tumors. Another two (cases 1 and 2) were negative for cytokeratin, excluding the tumor's epithelial origin. Immunochemistry stain that is positive for vimentin and negative for chromogranin and cytokeratin is suggestive of SCPT (Table 1)..$^{5}$

Surgical management includes complete resection of the involved pancreas, as was done in three of our patients. Had a surgical intervention been done in the first case, an attempt will have been made to resect the lymph nodes and involved areas of the liver. The role of neoadjuvant chemotherapy is described in case reports to decrease the size of the tumor before the surgery and in a patient with invasion of the superior mesenteric vein. ${ }^{2}$ However, it has also been reported that spontaneous regression of hepatic metastasis may occur in patients with SCPT. ${ }^{7}$

The long-term outcome of our patients is still unknown. All our patients are no longer on follow-up in our clinic. SCPT of the pancreas usually has a low malignant potential. Overall 5-year survival is approximately $97 \%$ among patients undergoing complete surgical resection. ${ }^{3}$ SCPT infiltration of the liver, stomach, and duodenum has been reported from 6 to $16 \%$ of cases, and about 10 to $15 \%$ may develop distant metastasis. ${ }^{7}$

In summary, we have discussed four Filipino children presenting with abdominal pain and palpable abdominal mass and were diagnosed with solid cystic pseudopapillary tumor, a rare pancreatic tumor. Surgical intervention is necessary as SCPT is a low-grade malignant tumor with a good prognosis.

\section{Statement of Authorship}

All authors participated in data collection and analysis, and approved the final version submitted.

\section{Author Disclosure}

All authors declared no conflicts of interest.

\section{Funding Source}

No funding support.

\section{REFERENCES}

1. Casadei R, Santini D, Calculli L, Pezzilli R, Zanini N, Minni F. Pancreatic Solid-Cystic Papillary Tumor: Clinical Features, Imaging Findings and Operative Management. JOP. 2006 Jan 11; 7(1): 137-44. PMID: 16407636.

2. Darius T, Brouwers J, VanDijck H, Bernard P. Solid and Cystic Papillary Neoplasm of the Pancreas: A Rare Tumor in Young Women. Acta Chirurgica Belgica, 2006; 106(6): 726-9. DOI: 10.1080/00015458.2006.11679994.

3. Zuriarrain A, Nir I, Bocklage T, Rajput A. Pseudopapillary Tumor of the Pancreas in a 17-Year-Old Girl. J Clin Oncol. 2011 May 10; 29(14):e395-6. DOI: 10.1200/JCO.2010.33.5364. Epub 2011 Feb 22. PMID: 21343547.

4. Guo N, Zhou Q, Chen R, Zou S, Li Z, Lin Q, et al. Diagnosis and surgical treatment of solid pseudopapillary neoplasm of the pancreas: analysis of 24 cases. Can J Surg. 2011 Dec; 54(6): 368-74. DOI: 10.1503/cjs.011810.

5. Mozaffar M, Abdollahi S. Acute post-traumatic presentation of a solid pseudopapillary tumor of the pancreas: A case report. Iranian Journal of Cancer Prevention, 2008 Dec; 1(1): 45-52.

6. Park JY, Kim SG, Park J. Solid pseudopapillary tumor of the pancreas in children: 15-year experience at a single institution with assays using an immunohistochemical panel. Ann Surg Treat Res, 2014 Mar; 86(3): 130-5. DOI: 10.4174/astr.2014.86.3.130.

7. Yoon HJ, Lim JH. Solid Pseudopapillary Tumor of the Pancreas with Hepatic Metastasis: Spontaneous Regression Over 10-year Followup Period. Korean J Radiol. 2012 Sep-Oct; 13(5): 648-51. DOI: 10.3348/kjr.2012.13.5.648. 